\title{
Observations of Solar Bursts Using the New Radio Spectrograph
}

\author{
A. Shanmugaraju ${ }^{1,2}, \mathrm{~S}$. Umapathy ${ }^{1}$ and V. Balasubramanian ${ }^{2}$ \\ ${ }^{1}$ School of Physics, Madurai Kamaraj University, Madurai 21, India. \\ ${ }^{2}$ Radio Astronomy Centre, TIFR, Ooty - 643 001, India.
}

\begin{abstract}
This paper presents a detailed analysis of 80 type III radio bursts, two noise storms and 10 type II radio bursts observed in the 30$80 \mathrm{MHz}$ range with the new radio spectrograph (Madurai Solar Radio Spectrograph) when it was operated on a daily basis during the periods December 1997 - April 1998 and August 1998. Results from statistical analysis of type III bursts are in agreement with earlier results. Employing a technique of separating the background continuum of noise storms show that the continuum is produced by electron accumulated from the preceding bursts. Also it is seen that the number of occurrences of type I bursts is maximum during the maximum of the background continuum. Comparison of records of type II bursts from MSRS with other spectrographs (Culgoora \& Hiraiso) for the same bursts indicate that the sensitivity of the MSRS at low frequencies is much better than the other spectrographs.
\end{abstract}

\section{Introduction}

For details of construction and the preliminary results obtained with the Madurai Solar Radio Spectrograph (MSRS), please refer to Shanmugaraju et al.(1999a).

\section{Results}

\subsection{Type III bursts}

Fig. 1 is a summary of results on drift rate, exciter velocity and starting frequency of the 80 type III bursts observed with the MSRS. It is seen that the drift rate is in the range $3-16 \mathrm{MHz} / \mathrm{s}$; exciter speed is about $20 \%$ of the velocity of light; the starting frequency of most bursts is at frequencies $\geq 80 \mathrm{MHz}$. These are in agreement with earlier results (McLean \& Labrum, 1985). The half power duration (hpd) of type IIIs measured at $50 \mathrm{MHz}$ is about 6-7sec.

\subsection{Noise Storms}

On two occassions (29 January 1998 and 15 February 1998) sustained noise storms with duration more than 3 hours were recorded. Continuum emission during these noise storms was separated by using a technique similar to that used by Malik \& Mercier (1996). Type I bursts were found to occur randomly during 

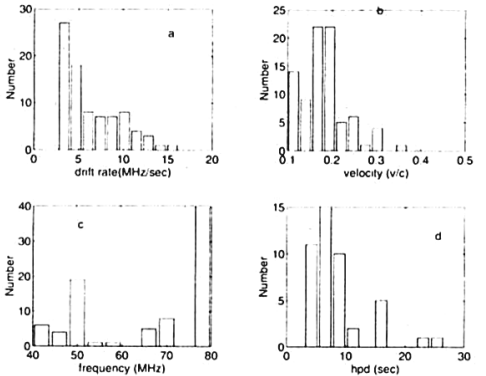

Figure 1. Distribution of $a$ ) drift rate, b) exciter velocity, c) starting frequency and d) half power duration (hpd) at $50 \mathrm{MHz}$ of the observed type III bursts.
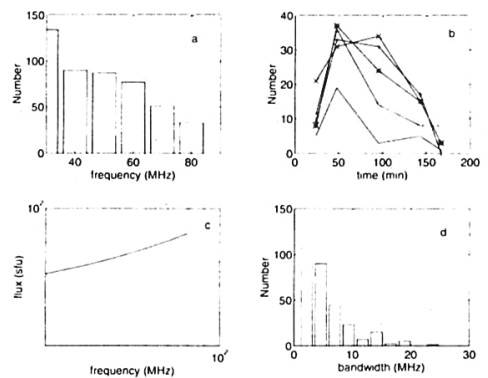

Figure 2. a) Histogram showing the number of bursts in each frequency range. b) Plot shows the time evolution of the bursts during storm. (o - $30 \mathrm{MHz}$, * - $40 \mathrm{MHz},+-50 \mathrm{MHz}, \times$ - $60 \mathrm{MHz}, . . \quad-70 \mathrm{MHz},-$ - $80 \mathrm{MHz}$ ). c) Flux density spectrum of the noise storm continuum observed on 29 Jan 1998. d) Distribution of the bandwidth of the storm bursts observed on 15 Feb 1998.

the storm continuum. However their rate of occurrence tends to increase during increase in the background continuum. It was also seen that continuum flux increases with the radio frequency of observation (Thejappa \& Kundu, 1991). Noise storm bursts (type I bursts) are found to have narrow bandwidths $(\leq 5$ $\mathrm{MHz}$ ) (see Fig.2).

\subsection{Type II Bursts}

The basic features of the type IIs observed with the MSRS are in agreement with earlier results (frequency drift rate in the range of $0.03-0.4 \mathrm{MHz} / \mathrm{s}$ ) and shock speed in the range $400-1000 \mathrm{~km} / \mathrm{s}$ ). These were of the average kind, i.e., not the strongest bursts. The emission at low frequencies for these bursts are more easily seen in the MSRS records than other spectrographs for the same bursts.

\section{References}

Malik, R.K. \& Mercier, C., 1996, Solar Phys., 165, 347.

McLean, D.J. \& Labrum, N.R., 1985, Solar Radiophy., Cambridge Univ. Press. Melrose, D.B., 1980, Solar Phys., 67, 357.

Shanmugaraju, A. et. al., 1999a, Solar Phys. 188, 155.

Thejappa, G. \& Kundu, M.R., 1991, Solar Phys., 132, 155. 